The Astronomical Journal, 128:1868-1871, 2004 October

(C) 2004. The American Astronomical Society. All rights reserved. Printed in U.S.A.

\title{
A POSSIBLE BROWN DWARF COMPANION TO THE WHITE DWARF GD 1400
}

\author{
J. FARIHI \\ Department of Physics and Astronomy, University of California, Los Angeles, CA 90095; jfarihi@astro.ucla.edu \\ AND \\ M. Christopher \\ Department of Astronomy, California Institute of Technology, Pasadena, CA 91125; mc@astro.caltech.edu \\ Received 2004 May 24; accepted 2004 July 1
}

\begin{abstract}
An unresolved, likely L dwarf companion to the DA white dwarf GD 1400 is reported. This would be only the second such system known, discovered 17 years after the prototype L dwarf, GD 165B, was determined to be a companion to a white dwarf. Photometric observations and model-predicted of the well-studied white dwarf primary stellar parameters indicate that GD 1400B has $J-K \gtrsim 2.0$ and $M_{K}=12.13 \mathrm{mag}$. If correct, this would place GD 1400B at spectral type L6, and it would be the lowest-luminosity unevolved companion known to a white dwarf and thus a definite brown dwarf. However, a low-resolution Keck NIRSPEC 2.1-2.4 $\mu$ m spectrum may not be consistent with known L dwarfs. Uncertainties in classification will remain until the binary is resolved or a trigonometric parallax is measured.
\end{abstract}

Key words: binaries: general — stars: individual (GD 1400) — stars: low-mass, brown dwarfs — white dwarfs

\section{INTRODUCTION}

In 1988, Becklin \& Zuckerman announced the discovery of the coolest dwarf star yet seen. Imaged with the first generation of infrared cameras, this companion to the white dwarf GD 165 went without proper classification for several years. The L dwarf prototype GD 165B came to represent the first new class of main-sequence stars in 100 years, a class believed to retain both stellar and substellar objects over the age of the Galaxy (Burrows et al.1997; Kirkpatrick et al. 1999, 2000; Burgasser et al. 2003). Since that time, more than 250 objects of this spectral type have been discovered. Rather ubiquitous in the field and in young clusters, L dwarfs have remained relatively infrequent companions to stars with $M \gtrsim$ $0.2 M_{\odot}$ (Zuckerman \& Becklin 1987, 1992; Schroeder et al. 2000; Oppenheimer et al. 2001; Hinz et al. 2002; Farihi et al. 2003; McCarthy \& Zuckerman 2004). Understanding the overall statistics and individual properties of the lowestmass companion systems is important for both star and planet formation.

This paper describes the properties of a very cool companion to the white dwarf GD 1400 (WD 0145-221, 01 $47^{\mathrm{m}} 21.8$, $-21^{\circ} 56^{\prime} 51^{\prime \prime} 4, \mathrm{~J} 2000$ ). Spectroscopic and photometric evidence is presented that indicates the presence of a low-mass, L-type dwarf within 0.3 of the primary.

\section{OBSERVATIONS AND DATA}

\subsection{V-Band Photometry}

Optical $V$-band data were taken 2004 January 3 with the CCD camera on the Nickel $1 \mathrm{~m}$ telescope at Lick Observatory. GD 1400 and a nearby Tycho-2 catalog star (TYC 4688-111-1) were both observed for a total of 3 minutes, each in three separate 1 minute exposures, yielding $\mathrm{S} / \mathrm{N} \sim 240$ on GD 1400 and $\mathrm{S} / \mathrm{N} \sim 7000$ on the $V=11.24$ mag calibrator (Høg et al. 2000; Bessell 2000). Conditions were clear but with poor seeing $(\theta \sim 4$ ".5) that appeared to remain relatively stable between target and calibrator observations. The individual frames were bias-subtracted, flat-fielded, registered, and then averaged to create a single reduced image on which to perform photometric measurements.

Photometry was executed with $10^{\prime \prime}$ and $20^{\prime \prime}$ diameter apertures on both GD 1400 and the calibrator, including telluric extinction corrections, producing consistent results to within $0.01 \mathrm{mag}$. The error in the measured flux of each object was less than $0.01 \mathrm{mag}$. The published uncertainty in the magnitude of TYC 4688-111-1 is $0.12 \mathrm{mag}$ (Høg et al. 2000). The result for GD 1400 is $V=14.85 \pm 0.12 \mathrm{mag}$.

\subsection{K-Band Spectroscopy}

GD 1400 was observed on 2004 January 16, using the Near Infrared Spectrograph (NIRSPEC; McLean et al. 1998) at Keck Observatory. NIRSPEC was used in low-resolution mode with a 0 ".76 slit, yielding a spectral resolution of $\lambda / \Delta \lambda \sim 1500$. The seeing was poor during the observations $(\theta \sim 1$ ". 2 at $K)$ and the telescope suffered from significant wind shake at the location of GD 1400, so no companion was resolved; nor was any elongation detected except due to the effects of wind shake.

Two 10 minute spectra (dithered by $15^{\prime \prime}$ for sky subtraction) were obtained in $K$ band using the N7 filter, which yielded a wavelength coverage of 2.04-2.45 $\mu \mathrm{m}$. The observations had a fixed position angle on the sky of $38^{\circ}$. HD 63586, an A0 V star, was observed at similar air mass for atmospheric calibration.

The spectrum of GD 1400 was reduced using the REDSPEC data reduction package. The images were cleaned of bad pixels, flat-fielded, pair-subtracted, and spatially rectified. Two spectra of GD 1400 were extracted, averaged, divided by the calibrator spectrum (scaled by a blackbody), wavelength-calibrated, and normalized. The average of the two spectra is shown in Figure 1.

\section{RESULTS}

An unresolved companion was suspected based on the optical and near-infrared data available on GD 1400. In the 


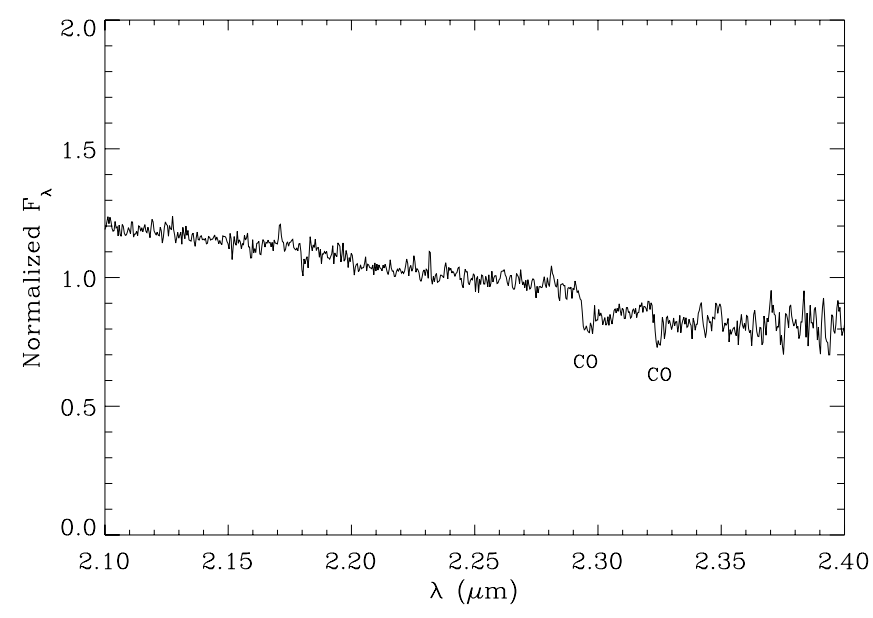

FIG. 1.-Combined spectrum of GD 1400 and L dwarf companion, normalized to 1.0 at $2.235 \mu \mathrm{m}$.

following sections, evidence is presented that reveals a lowmass companion causing excess emission at $2.2 \mu \mathrm{m}$.

\section{1. $J$ and $K$ Magnitudes and Color}

There exist two independent spectroscopic analyses of the hydrogen-rich (DA) white dwarf GD 1400. Both Koester et al. (2001) and Fontaine et al. (2003) give results based on high-resolution spectroscopy that agree quite well: $T_{\text {eff }}=$ $11,605 \mathrm{~K}, \log g=8.05$ and $T_{\text {eff }}=11,550 \mathrm{~K}, \log g=8.14$, respectively. The average and standard deviation of these two results $\left(T_{\text {eff }}=11,580 \pm 40 \mathrm{~K}, \log g=8.10 \pm 0.06\right)$ were used to predict model colors and absolute magnitudes, with errors, for GD 1400 (Bergeron et al. 1995a).

Before considering distances and absolute magnitudes for GD 1400B, the flux ratio of primary and secondary should be considered. Based on the measured $V$ magnitude and the $V-K=-0.24$ model-predicted color for GD 1400, the predicted $K$ magnitude is $K=15.09 \pm 0.12$ mag. (The modelpredicted color has less than 0.01 mag uncertainty and is essentially independent of surface gravity, so that the error is all measurement error in V.) The Two Micron All Sky Survey (2MASS) point-source catalog gives a $\mathrm{S} / \mathrm{N}=19.2$ measurement of $K_{s}=14.34 \pm 0.06 \mathrm{mag}$ for the composite pair. Ignoring corrections between $2.2 \mu \mathrm{m}$ filter sets, a deconvolved magnitude of $K=15.10 \pm 0.20 \mathrm{mag}$ is calculated for the companion. If the white dwarf models are accurate, the companion and primary appear equally luminous at this wavelength.
There is little or no excess emission measured for GD 1400 at $1.2 \mu \mathrm{m}$, which is consistent with a very red color of $J-K \gtrsim 2.0$ for its companion (Table 1 ) and consistent with a spectral type of L5.5 or later (Kirkpatrick et al. 2000; Dahn et al. 2002). Within the uncertainties of the 2MASS measured $J$ magnitude of the composite and the white dwarf model extrapolation, it is possible that the companion $J$-band flux is undetected.

The radius of GD 1400, which has been determined spectroscopically by two independent measurements, can be used to estimate an absolute magnitude for GD 1400B. At an assumed 39 pc photometric distance for GD 1400 (based on the model predicted absolute magnitude of $M_{V}=11.88 \pm$ $0.10 \mathrm{mag}$ for the white dwarf) an absolute $K$ magnitude of $M_{K}=12.13 \pm 0.22 \mathrm{mag}$ is determined for the $\mathrm{L}$ dwarf companion. These data are most compatible with spectral type L6 and $T_{\text {eff }} \sim 1650 \mathrm{~K}$ for GD 1400B (Reid et al. 1999; Kirkpatrick et al. 2000; Dahn et al. 2002).

\subsection{A Comparison with the GD 165 Binary System}

Coincidentally, the GD 165 system (DA4.2+dL4), which has a white dwarf primary with similar temperature and surface gravity to GD 1400, serves as a useful guide and point of comparison for the following analysis. There are two independent spectroscopic parameter determinations for GD 165. Both Koester et al. (2001) and Bergeron et al. (1995b) give similar results: $T_{\text {eff }}=11,970 \mathrm{~K}, \log g=7.91$ and $T_{\text {eff }}=11,980 \mathrm{~K}$, $\log g=8.06$, respectively. In addition, GD 165 has a trigonometric parallax that places it at $31.5 \mathrm{pc}$. The difference between the model-predicted versus measured absolute magnitudes for GD $165 \mathrm{~A}$ are $0.06 \mathrm{mag}$ at $V, 0.03 \mathrm{mag}$ at $J$, and $0.04 \mathrm{mag}$ at $K$ (Bergeron et al. 1995a, 1995b; Becklin \& Zuckerman 1988). Hence, the models appear quite accurate in this case.

In contrast to the equally luminous components of the GD 1400 system, GD 165B is 0.41 mag brighter than its white dwarf primary at $K$. Provided that brown dwarfs and the lowest-mass stars all have radii near $1 R_{\mathrm{J}}$ after $1 \mathrm{Gyr}$ (Burrows et al. 2001), GD 1400B appears cooler than the L4 dwarf GD 165B. The total $J$-band flux of the GD 1400 system is consistent (within the errors) with a single star; yet the combined $J$-band flux of both components of the GD 165 system is 0.29 mag brighter than the flux from the white dwarf alone (Bergeron et al. 1995a). Again, if the white dwarf and brown dwarf models used here are accurate, GD 1400B appears to be cooler than GD 165B.

Although this photometric evidence points to an object cooler than GD 165B, there is still room for some error in the models and perhaps even in the spectroscopy of the primary

TABLE 1

Magnitudes for GD 1400 and Companion

\begin{tabular}{|c|c|c|c|c|}
\hline Filter & $\begin{array}{c}\lambda_{0} \\
(\mu \mathrm{m})\end{array}$ & $\begin{array}{l}\text { Composite } \\
\text { (mag) }\end{array}$ & $\begin{array}{l}\text { White Dwarf } \\
\text { (mag) }\end{array}$ & $\begin{array}{l}\text { L Dwarf } \\
\text { (mag) }\end{array}$ \\
\hline$V \ldots \ldots \ldots \ldots \ldots \ldots \ldots \ldots$ & 0.55 & $14.85 \pm 0.12$ & $14.85 \pm 0.12$ & \\
\hline 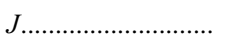 & 1.22 & $14.92 \pm 0.03$ & $15.02 \pm 0.12$ & $17.5 \pm 0.9^{\mathrm{a}}$ \\
\hline 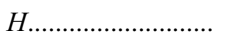 & 1.65 & $14.45 \pm 0.05$ & $15.02 \pm 0.12$ & $15.42 \pm 0.26$ \\
\hline K & 2.16 & $14.34 \pm 0.06$ & $15.09 \pm 0.12$ & $15.10 \pm 0.20$ \\
\hline
\end{tabular}

Notes.-Measured magnitudes for the composite star are from this work and 2MASS (the $2.2 \mu \mathrm{m}$ data point is a $K_{s}$ magnitude). Predicted near-infrared magnitudes for the white dwarf are from Bergeron et al. (1995a).

a The deconvolved L dwarf $J$ magnitude is extremely sensitive to small changes in the measured and predicted magnitudes ( $(3.1)$. 


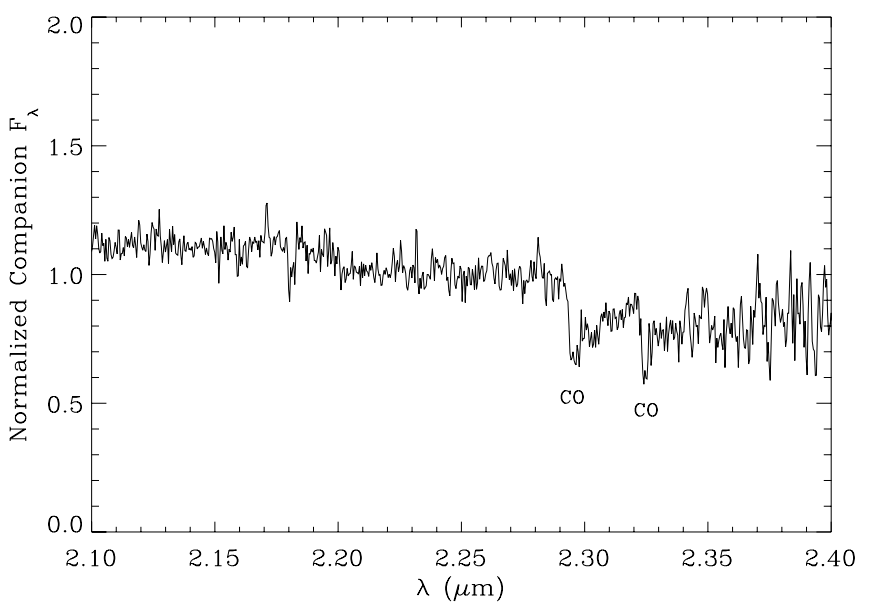

FIG. 2.-L dwarf companion spectrum after subtraction of an $11,500 \mathrm{~K}$ blackbody of equal flux at $2.2 \mu \mathrm{m}$. The $\mathrm{CO}$ band heads at 2.29 and $2.32 \mu \mathrm{m}$ are clearly seen. Also note the absence of $\mathrm{Na}$ at $2.21 \mu \mathrm{m}$. The standard deviation between 2.07 and $2.28 \mu \mathrm{m}$ is 0.06 flux units per resolution element.

white dwarf. The GD 1400 system is unresolved, and there currently exists no trigonometric parallax.

\subsection{K-Band Spectrum}

Near-infrared spectroscopy was performed to examine the possibility of a red background object or dust as the cause of the excess emission seen at $2.2 \mu \mathrm{m}$. The $2.1-2.4 \mu \mathrm{m}$ spectrum of GD 1400 is shown in Figure 1. The two features seen at 2.29 and $2.32 \mu \mathrm{m}$ are $\mathrm{CO}$ band heads, which are indicative of a low-mass companion and readily visible in this composite spectrum. An 11,500 K blackbody spectrum, scaled to have exactly half the flux of the composite at $2.20 \mu \mathrm{m}$, was subtracted from the spectrum in Figure 1, thus effectively removing the expected contribution from the featureless white dwarf at these wavelengths. The resulting spectrum is shown in Figure 2.

In the subtracted spectrum, both $\mathrm{CO}$ band heads have $\mathrm{S} / \mathrm{N}>25$. Figure 3 shows the equivalent widths (EWs) of these features for several $\mathrm{M}$ and $\mathrm{L}$ dwarfs from Cushing (2000) together with GD 1400B - all measured in a uniform way for this work. The EWs for GD 1400B appear stronger than those of known single $\mathrm{L}$ and $\mathrm{M}$ dwarfs. Oversubtraction of the white dwarf continuum from the composite spectrum could, in principle, cause the measured $\mathrm{CO}$ band head EWs to be larger than their intrinsic values. Another possibility is close binary interactions, such as heating of the companion by the white dwarf. Further observations are necessary to reconcile this apparent anomaly.

There is an absence of $\mathrm{Na}$ at $2.21 \mu \mathrm{m}$, which can be seen weakly as late as spectral type L2, but not later. EWs vary between 0.2 and $3.0 \AA$ for early L dwarfs (McLean et al. 2003; Cushing 2000). A measurement of this region in the spectrum of GD 1400B is consistent with an EW of less than $1.0 \AA$. The noise in the region from 2.19 to $2.23 \mu \mathrm{m}$ is 0.040 flux units per resolution element, and the Na line typically has a full width of $\sim 50 \AA$ (McLean et al. 2003; Cushing 2000 ) in late $M$ dwarfs, or roughly five resolution elements of 2.6 pixels each. Hence, an EW as small as $1.0 \AA$ could have been detected at the $1 \sigma$ level. The lack of $\mathrm{Na}$ is inconsistent with an M dwarf.

The conclusion based on spectroscopy alone is that an early L type cannot be ruled out with confidence. Although the EWs

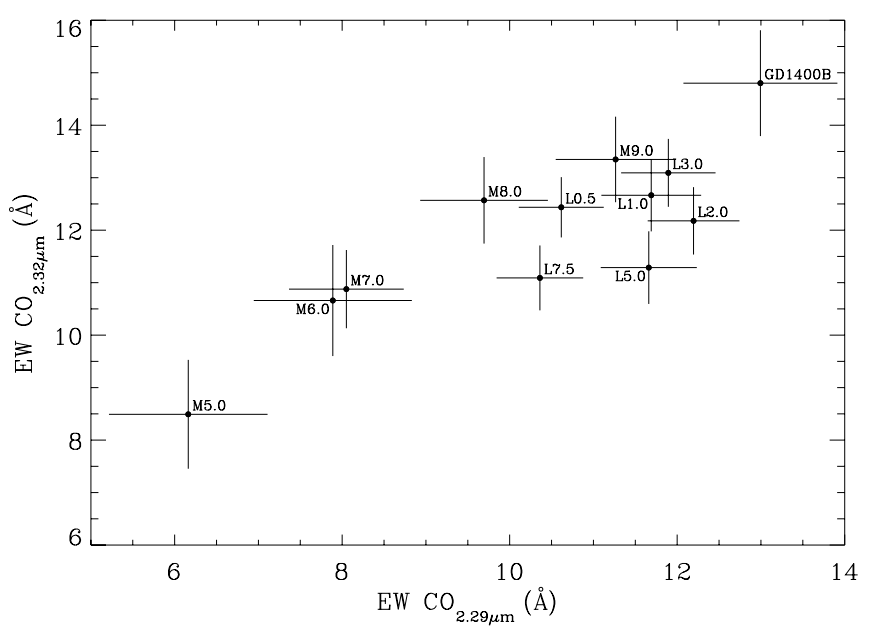

FIG. 3.-EWs measured for individual $\mathrm{M}$ and $\mathrm{L}$ dwarfs (from Cushing 2000). GD 1400B appears to be somewhat anomalous.

of the first and second $\mathrm{CO}$ band heads are larger than those seen in single $\mathrm{L}$ and late $\mathrm{M}$ dwarfs, this could be due to close binary interactions or model uncertainties that led to oversubtraction of the white dwarf flux. The fact that $\mathrm{Na}$ is not manifest in the spectrum may be at odds with the CO EWs, but it is consistent with the photometry.

\section{DISCUSSION}

\subsection{The Age and Mass of GD $1400 B$}

GD 1400, a $0.67 M_{\odot}$ white dwarf, has been cooling for 0.51 Gyr according to models ( $\mathrm{P}$. Bergeron 2002, private communication). From the initial to final mass relation for white dwarfs (Weidemann 1987, 1990, 2000; Bragaglia et al. 1995), a white dwarf with this mass has evolved from a mainsequence progenitor with a mass in the range 2.5-3.0 $\mathrm{M}_{\odot}$. The main-sequence lifetime then falls within the range $0.5-$ 1.0 Gyr (Maeder 1989), yielding a total age between 1.0 and 1.5 Gyr. The very moderate proper motion of GD 1400 , $\mu \approx 0$ ".05 $\mathrm{yr}^{-1}$ (Zacharias et al. 2004), also supports the conclusion that this is a relatively young system. Assuming $v_{\text {rad }}=0$, its Galactic space velocity relative to the LSR is $(U, V, W)=(-4,+5,+9) \mathrm{km} \mathrm{s}^{-1}(U$ is taken to be positive toward the Galactic anticenter, $V$ positive in the direction of Galactic rotation, and $W$ positive toward the north Galactic pole), with a total space motion of only $9 \mathrm{~km} \mathrm{~s}^{-1}$ with respect to the Sun - which is typical of young disk objects (Mihalas \& Binney 1981; Leggett 1992). At $1.25 \mathrm{Gyr}$, a $T_{\text {eff }} \sim 1650 \mathrm{~K}$ brown dwarf is predicted to have $M \approx 60 M_{\mathrm{J}}$ (Chabrier et al. 2000).

\subsection{Origin and Evolution of the GD 1400 Binary System}

The orbital separation of GD $1400 \mathrm{~B}$ is uncertain. The system remained unresolved in subsequent NIRSPEC $K$-band images taken two weeks after the spectrum was obtained. The image with the best spatial resolution reveals a full width of $\theta=0.82$ and a sufficiently symmetric PSF to rule out an equally luminous binary with separation $\gtrsim 0$ "'3. Although there is the possibility of a chance alignment, the most likely explanation for the unresolved nature of the binary is a relatively small, $a<12$ AU, semimajor axis.

During the AGB phase of stellar evolution, orbiting bodies outside of $\sim 5$ AU should eschew the expanding envelope and eventually have their semimajor axes expanded by a factor 
(typically 3-5) related to the mass lost (Jeans 1924; Zuckerman $\&$ Becklin 1987). Inside of $\sim 5 \mathrm{AU}$, a companion should spiral in due to tidal and frictional interaction with the slow AGB wind and expanding photosphere (Debes \& Sigurdsson 2002). Hence, it is likely that the semimajor axis of GD 1400B's orbit is less than a few astronomical units. A sensitive search for radial velocity variations might be able to confirm or rule out a close binary system.

\section{CONCLUSION}

Based on photometric data presented here, the first unambiguous substellar companion to a white dwarf may have been discovered within 0"3 ( 12 AU) of GD 1400 (DA4.4). However, the near-infrared spectroscopy may indicate a $\mathrm{CO}$ temperature that is higher than the photospheric temperature indicated by the photometry. This could be the result of close binary interactions causing the $\mathrm{CO}$ band heads to appear more strongly than in isolated L dwarfs, or it may be due to errors in the models used to remove the flux of the primary.

From a direct comparison with the GD 165 binary system (relying on the same white dwarf and brown dwarf models), it appears that GD $1400 \mathrm{~B}$ is cooler than spectral type L4. The deconvolved magnitudes and colors of GD 1400B are most consistent with spectral type L6, indicative of a $60 M_{\mathrm{J}}$ brown dwarf at an estimated age of 1.25 Gyr. Its true nature and origins will remain uncertain until more is known about the binary system. A trigonometric parallax determination, radial velocity measurements, or very high resolution imaging should be able to further constrain the properties of this system.

Both authors owe a debt of gratitude to M. Cushing for kindly providing us with his spectra for analysis here and to N. Scoville for donating some of his Keck NIRSPEC time to observe GD 1400. J. F. wishes to express sincere thanks to B. Zuckerman for his assistance in acquiring the optical data, to E. Gates of Lick Observatory for taking the optical data, to L. Prato and M. McGovern for securing follow-up nearinfrared images and many helpful discussions, and to E. E. Becklin and B. Zuckerman for careful readings of the manuscript and constructive comments. Some of the data presented herein were obtained at Keck Observatory, which is operated as a scientific partnership among the California Institute of Technology (Caltech), the University of California, and the National Aeronautics and Space Administration (NASA). Some data used in this paper are part of the Two Micron All Sky Survey, a joint project of the University of Massachusetts and the Infrared Processing and Analysis Center (IPAC)/Caltech, funded by NASA and the National Science Foundation (NSF). 2MASS data were retrieved from the NASA/IPAC Infrared Science Archive, which is operated by the Jet Propulsion Laboratory, Caltech, under contract with NASA. J. F. has been supported in part by grants from NASA to UCLA and M. C. by NSF grant AST 02-28955.
Becklin, E. E., \& Zuckerman, B. 1988, Nature, 336, 656

Bergeron, P., Wesemael, F., \& Beauchamp, A. 1995a, PASP, 107, 1047

Bergeron, P., Wesemael, F., Lamontagne, R., Fontaine, G., Saffer, R. A., \& Allard, N. F. 1995b, ApJ, 449, 258

Bessell, M. S. 2000, PASP, 112, 961

Bragaglia, A., Renzini, A., \& Bergeron, P. 1995, ApJ, 443, 735

Burgasser, A. J. 2003, ApJ, 592, 1186

Burrows, A., et al. 1997, ApJ, 491, 856

Burrows, A., Hubbard, W. B., Lunine, J. I., \& Liebert, J. 2001, Rev. Mod. Phys., 73, 719

Chabrier, G., Baraffe, I., Allard, F., \& Hauschildt, P. 2000, ApJ, 542, 464

Cushing, M. 2000, Ph.D. thesis, Univ. Hawaii

Dahn, C. C., et al. 2002, AJ, 124, 1170

Debes, J. H., \& Sigurdsson, S. 2002, ApJ, 572, 556

Farihi, J., Becklin, E. E., \& Zuckerman, B. 2003, in IAU Symp. 211, Brown Dwarfs, ed. E. Martín (San Francisco: ASP), 289

Fontaine, G., Bergeron, P., Billères, M., \& Charpinet, S. 2003, ApJ, 591, 1184

Hinz, J., McCarthy, D., Simons, D., Henry, T., Kirkpatrick, J., \& McGuire, P. 2002, AJ, 123, 2027

Høg, E., et al. 2000, A\&A, 355, L27

Jeans, J. H. 1924, MNRAS, 85, 2

Kirkpatrick, J. D., Allard, F., Bida, T., Zuckerman, B., Becklin, E. E., Chabrier, G., \& Baraffe, I. 1999, ApJ, 519, 834

\section{REFERENCES}

Kirkpatrick, J. D., et al. 2000, AJ, 120, 447

Koester, D., et al. 2001, A\&A, 378, 556

Leggett, S. K. 1992, ApJS, 82, 351

Maeder, A. 1989, in Astrophysical Ages and Dating Methods, ed. E. VangioniFlam, M. Cassé, J. Audouze, \& J. Trân Thanh Vân (Gif-sur-Yvette: Ed. Frontières), 71

McCarthy, C., \& Zuckerman, B. 2004, AJ, 127, 2871

McLean, I. S., et al. 1998, Proc. SPIE, 3354, 566

McLean, I. S., McGovern, M. R., Burgasser, A. J., Kirkpatrick, J. D., Prato, L., \& Kim, S. S. 2003, ApJ, 596, 561

Mihalas, D., \& Binney, J. 1981, in Galactic Astronomy: Structure and Kinematics (San Francisco: Freeman), 278

Oppenheimer, B., Golimowski, D., Kulkarni, S., Matthews, K., Nakajima, T., Creech-Eakman, M., \& Durrance, S. 2001, AJ, 121, 2189

Reid, I. N., et al. 1999, ApJ, 521, 613

Schroeder, D., et al. 2000, AJ, 119, 906

Weidemann, V. 1987, A\&A, 188, 74 - 1990, ARA\&A, 28, 103 2000, A\&A, 363, 647

Zacharias, N., et al. 2004, AJ, 127, 3043

Zuckerman, B., \& Becklin, E. E. 1987, ApJ, 319, L99 . 1992, ApJ, 386, 260 